\title{
Processus de structuration du contrôle de gestion dans les pme : Une analyse en contexte camerounais
}

\author{
Nocheh Nselapi Dieudonné ${ }^{1}$; Teuguia Tadjuidje Gilles ${ }^{2}$ and Tchatchoua Thierry Roméo $^{3}$ \\ 1,2 Finance-Comptabilité - FSEGA- Université de Douala - Cameroun \\ ${ }^{3}$ Marketing et Organisation - FSJP- Université de Douala - Cameroun
}

\begin{abstract}
Résumé : Les PME en phase de développement sont exposées à deux catégories de risque à savoir le risque entrepreneurial et le risque managérial. Ce dernier est lié à l'informalité de la gestion de celle-ci. Dans les Pme camerounaises, on note une mauvaise organisation des activités, l'absence d'un système d'information et la déficience en outils permettant de mieux prévoir et d'assurer le pilotage de l'entreprise. Face à cela, l'opportunité de passer des leviers informels de contrôle vers des systèmes de contrôle formels en occurrence le contrôle de gestion (CDG) constitue une phase décisive pour les PME en quête de performance. L'objectif de l'étude est d'identifier les étapes de structuration du CDG dans les PME en vue d'amorcer la phase de croissance. De ce fait, une étude a été menée auprès de huit (08) PME camerounaises et l'analyse du contenu thématique de chaque cas révèle que la structuration du CDG dans les PME est un processus qui s'effectue par étape successive. II ressort des analyses que trois types de processus caractérisent l'organisation formelle de la PME à savoir le processus progressif, le processus stationnaire et le processus dit bloquer. Aussi, l'importance de la structuration du CDG doit s'inscrire dans une dynamique de type projet et privilégier une gestion par la compétence au détriment d'une gestion dite familiale.
\end{abstract}

Mots-clés : Processus, Structuration, Contrôle de gestion, PME camerounaise.

\begin{abstract}
SMEs in the development phase are exposed to two categories of risk such as entrepreneurial risk and managerial risk. The latter is linked to the informality of its management. In Cameroonian SMEs, we note a poor organization of activities, the limited information system and the lack of tools to better forecast and ensure the management of the company. Faced with this, the opportunity to move from informal control levers to formal control systems, in this case management control (CDG), constitutes a decisive phase for SMEs in search of performance. The objective of the study is to identify the stages in the structuring of management control in SMEs in order to initiate the growth phase. As a result, a study was carried out among eight (08) Cameroonian SMEs and the analysis of the thematic content of each case reveals that the structuring of CDG in SMEs is a process that takes place in successive stages. It emerges from the analyzes that three types of process characterize the formal organization of the SME, namely the progressive process, the stationary process and the so-called blocking process. Also, the importance of structuring the CDG must be part of a project-type dynamic and favor management by competence.
\end{abstract}

Keywords: Process - Structuring - Management Control- Cameroonian SME. 


\section{Introduction}

Les PME durant leur cycle de vie sont confrontées à deux moments majeurs à savoir la phase de création et la phase de croissance. Durant la première phase, il faut redéfinir le produit et positionner l'offre dans un contexte concurrentiel. Par contre au cours de la phase de croissance, elle éprouve la nécessité de bien organiser les activités, de disposer d'un système d'information, bien exploiter les ressources humaines et matérielles d'où l'implémentation d'un dispositif de contrôle de gestion (désormais CDG).

Les recherches sur le CDG dans les PME ont suivi plusieurs phases distinctes. Les premiers travaux sur le CDG dans les PME ont porté principalement sur les instruments utilisés en matière de CDG. Ils ont été consacrés à l'étude de l'existence et l'usage des outils de CDG dans les PME (Marchesnay, 1995 ; Fernandez et al. 1996 ; Bergeron, 2000, Ngongang 2013, Mayegle et al, 2016).

Au-delà des conclusions favorables, plusieurs auteurs ont émis des réserves quant à la pertinence des résultats obtenus du fait du caractère déclaratif des données (Nobre ; 2001 ; Marchesnay et Fourcade, 1997). Ce qui a remis en cause les résultats obtenus sur les pratiques du CDG dans les PME. L'approche instrumentale se trouve ainsi limitée dans les recherches visant à comprendre les pratiques du CDG des PME. Selon ces auteurs, il ne s'agit plus de recenser les outils mobilisés par les PME ou de dire si les PME pratiquent le CDG ou pas. II s'agit plutôt d'étudier comment les systèmes de CDG apparaissent et évoluent dans l'entreprise. Selon Meyssonnier et Zawadzki (2008), l'axe d'analyse de la structuration du CDG dans les PME est une étape très importante et essentielle à leur survie.

L'objectif de ce papier est de caractériser le processus de structuration du CDG dans les PME. Pour y parvenir, une interaction est nécessaire avec les acteurs concernés. Nous passons d'abord en revue les mécanismes de passage des leviers informels au contrôle de gestion dans les PME, ensuite la description de la démarche méthodologique mobilisée de travail et enfin la présentation les résultats et recommandations.

\section{Les étapes de structuration du CDG dans les PME : Un état de l'art}

Le contrôle de gestion dans sa mission première consiste à apprécier le degré et la manière d'atteinte des objectifs assignés. Il s'est élargi par la suite comme un moyen utilisé par les dirigeants pour agir sur des comportements des acteurs du système. Pour Simons (1995), le CDG est l'ensemble des processus et procédures basé sur l'information utilisée par les gestionnaires pour adapter certaines configurations des activités de l'entreprise. Ce mode de contrôle est intentionnel dans le sens où c'est l'entreprise qui décide d'écrire ses règles de fonctionnement, de définir les outils qu'elle va mobiliser pour suivre son activité. L'entrepreneur peut décider d'instituer un contrôle formel au sein de son entreprise ou poursuivre avec un contrôle informel.

\subsection{Le passage du système informel au système formel de contrôle}

Dans les PME en création et surtout familiale, la coordination des comportements repose sur un management de type clanique selon lequel la socialisation et les intérêts des individus s'alignent avec ceux de l'organisation (Langevin et Naro, 2003). Le contrôle social semble convenablement atténuer l'inexistence de la fonction CDG dans l'entreprise et le besoin d'introduire un système global et cohérent de management de la performance n'y est pas ressenti. Les responsables se satisfont des mécanismes de coordination informels mis en œuvre. Dans cette perspective d'exploration, les 
promoteurs n'y trouvent généralement pas d'inconvénient. La coordination informelle des comportements dans l'entreprise repose sur des aspects liés aux acteurs composant l'organisation à travers la mise en place d'une culture particulière. Dans le cas de la culture clanique, l'on retrouve un esprit familial diffusé par le dirigeant et celui-ci entretient des relations particulières avec les salariés. La confiance joue un rôle majeur dans le maintien d'une certaine harmonie dans l'entreprise. Pour certains auteurs, la confiance peut être similaire à un système informel de contrôle des comportements. Cette forme de coordination est plus présente dans les PME à forte connotation familiale, les activités sont coordonnées par des représentants informels communs et une confiance mutuelle. En plus de la confiance, Dupuy et al. (1989) font référence à la convention en tant que moyen de coordination. La théorie des conventions, émerge dans les années 80 . Elle se distingue de l'approche économique pure, de la grande analyse de la coordination et de l'accord en entreprise. Selon Gomez (1996), dans un contexte d'incertitude, c'est davantage par une approche mimétique que les acteurs optent pour la convention. Cette coordination par la convention s'appuie sur l'idée de "rationalité mimétique" (Montmorillon, 1999). Le recours à ce mécanisme a l'avantage de permettre une autorégulation et une auto-organisation de l'entreprise dont l'objectif est d'assurer sa bonne marche. Par conséquent, la convention représente une perspective d'analyse de la coordination dans les organisations informelles. La coordination des comportements à travers des mécanismes informels et interindividuels (leadership, contexte émotionnel, culture, ...) présentent des insuffisances dans les PME en cas d'évolution structurelle (augmentation de sa taille, départ d'un leader, ...). L'organisation se trouve dans l'incapacité à la faveur des seuls leviers informels de contrôle de maîtriser les comportements en son sein (Zawadzki, 2009). L'entreprise dans une configuration de cette nature se trouve inexorablement face à un dilemme de poursuivre avec ces pratiques informelles qui l'éloignent parfois de son objectif et mettent en péril sa survie; ou alors modifier sa configuration organisationnelle en mettant en place des leviers formels de contrôle à l'instar du CDG. Il s'agit d'une étape transitoire très délicate et critique à laquelle l'entreprise est appelée à faire face.

La pérennité de la Pme passe par la mise en œuvre d'un dispositif de CDG formel. Cet outil de gestion permet à l'entreprise de définir ses objectifs de performance à atteindre, de les suivre au quotidien, et de se rassurer que les comportements des différents acteurs à la performance concourent à l'objectif commun.

Au Cameroun, Mayegle et Ngo-Nguidjol (2016) ont réalisé une enquête sur un échantillon de 232 PME. Les résultats montrent que $83,6 \%$ de PME de l'échantillon disposent des outils de CDG. Ces statistiques corroborent celles obtenues par Ngongang (2013) réalisées auprès de 68 entreprises de taille comprise entre 1 et plus de 100. Il relevait déjà la présence de plusieurs outils de CDG dans les PME camerounaises à savoir les budgets, la comptabilité analytique, les tableaux de bord, le calcul des écarts et des coûts. II faisait une observation importante selon laquelle l'intérêt pour les outils de CDG dans les PME camerounaises est très faible. Toutefois, il convient d'observer que les travaux dans différents contextes tant camerounais qu'ailleurs ont mis l'accent sur les outils de contrôle de gestion manipulés par les PME. Toutefois, au-delà des conclusions favorables obtenues quelques fois, plusieurs de ces auteurs ont émis des réserves quant à la pertinence de ces résultats obtenus du fait du caractère déclaratif des données (Nobre (2001). Ce qui remet nécessairement en cause les résultats obtenus sur les pratiques du CDG dans les PME et interpelle davantage sur l'étude du processus de structuration de cette fonction dans les entreprises de cette taille. 


\subsection{La structuration du contrôle de gestion dans les PME : une analyse à partir de la théorie de la traduction}

La traduction est un processus qui permet d'établir un lien clair entre des activités hétérogènes au sein d'une organisation. Chaque étape prend des concepts qui sont de nombreuses caractéristiques du processus de traduction. La théorie de la traduction permet d'examiner le parcours des nouveaux outils qui sont introduits auprès des acteurs dans l'organisation à différents moments qui composent les étapes de traduction. La convergence des traductions faites par les acteurs majeurs, en particulier les porte-paroles légitimes, conditionne la réussite de l'innovation. Différents participants traduisent les outils afin qu'ils puissent répondre à leurs besoins respectifs ou leur permettre de conserver leur position et leur influence dans l'organisation (Alcouffe et al., 2008). Le traducteur joue un rôle déterminant dans ce sens que son intervention doit aboutir à l'alignement des traductions des différents réseaux pour que les outils soient acceptés.

Tableau n ${ }^{\circ} 01$ : La synthèse des étapes de la traduction

\begin{tabular}{|c|ll|}
\hline $\begin{array}{c}\text { La problématisation } \\
\text { ou « l'art de se rendre } \\
\text { indispensable » }\end{array}$ & $\begin{array}{l}\text { - } \begin{array}{l}\text { Proposer un mode virtuel au sein duquel l'artefact technologique devient } \\
\text { indispensable }\end{array} \\
\text { - }\end{array}$ & $\begin{array}{l}\text { Identifier les alliances à sceller } \\
\text { - Identifier un ou des points de passage obligé }\end{array}$ \\
\hline L'intéressement & - & Concrétiscr le réscau d'alliances cnvisagé \\
& - Traduire des identités, déplacer des alliances pour en former des nouvelles \\
\hline L'enrôlement & - & Affecter des actants à un rôle précis \\
\hline $\begin{array}{c}\text { La mobilisation et le } \\
\text { rallongement }\end{array}$ & - & Stabiliser le réseau par la représentativité suffisante de ses membres \\
\hline
\end{tabular}

Source : Synthèse des travaux de Callon (1986), Akrich et al. (1988)

A travers les quatre étapes de la traduction, un réseau forme, l'étape de la problématisation aboutit à la création d'un réseau de liens contraignants. Un " acteur - réseau » se dessine, en d'autres termes un réseau agissant comme un acteur disposant de façon conciliante ou contraignante les autres acteurs pour qu'ils tiennent ensemble, et essayant de connecter les différents alliés ensemble et rendre l'asymétrie irréversible.

Dans le cadre de cette recherche, le concept de traduction se réfère à la définition de Barbelivien et Meyssonnier (2018) pour qui : "la structuration du CDG est un processus, un continuum d'états successifs de développement des pratiques de CDG entre un point de départ, où la fonction CDG est inexistante et les outils de CDG sont épars et servent juste à améliorer la prise de décision du dirigeant, et un point d'arrivée, avec une fonction de contrôleur de gestion légitime et des dispositifs inscrits au cœur du fonctionnement de l'entreprise (prévision budgétaire, reporting mensuel, contrôle budgétaire, système d'incitation des managers articulé avec les objectifs managériaux de performance, etc.)»

De l'analyse de cette définition, on déduit que la structuration du CDG revêt deux perspectives à savoir une perspective temporelle et une perspective instrumentale. La première recouvre la dynamique processuelle de la démarche de formalisation du CDG. La seconde désigne la conception et l'implantation de nouveaux outils de gestion dans l'entreprise. 
De ce qui précède, il se dégage que la structuration du CDG consiste en la formalisation progressive de la fonction CDG dans l'entreprise. Mieux encore la structuration du CDG renvoie à l'introduction du CDG et son évolution dans l'entreprise. L'introduction du CDG dans la PME ne sous-entend pas que la fonction est complète, mais une tentative d'introduction des outils de pilotage. Au fil des années avec l'évolution de l'entreprise et de ses impératifs, l'entreprise va faire évoluer cette fonction pour qu'elle s'adapte à ses besoins. En effet, initialement comme le relève Barbelivien et Meyssonnier (op cité), la fonction est quasi absente et les outils très rudimentaires, mais progressivement que l'entreprise gagne en maturité ou évolue, elle améliore son dispositif de CDG jusqu'à intégration en son sein d'une fonction de CDG. La structuration du CDG est donc de deux ordres, l'un instrumental et l'autre temporel. Selon les travaux de Barbelivien (2016), Barbelivien et Meyssonnier (2018) la structuration du CDG dans les PME obéit à une démarche par étape et graduelle de remise cause d'une situation insatisfaisante, de modification dans la structure et d'amélioration dans l'instrumentation de gestion.

La structuration du CDG au regard de la théorie de la structuration se fait par étape. II s'agit du passage d'une situation de contrôle essentiellement informel vers une autre où l'entreprise développe un dispositif de gestion formel. La théorie de la traduction permet de mettre en évidence les étapes du processus à savoir une étape de problématisation, suivie d'une étape d'intéressement, ensuite une étape d'enrôlement et une dernière étape de mobilisation. Comment les Pme camerounaises procèdent-elle pour structurer leur CDG ?

\section{La Présentation de la démarche méthodologique de l'étude}

La particularité de la technique de collecte des données dans cette étude tient à la simultanéité et la spontanéité des opinions du répondant dans la phase initiale. A l'opposé de démarche classique où la collecte des données s'effectue une seule fois, nous avons effectué des allers et retours sur le terrain pour observer des comportements des acteurs et collecter les données nécessaires à la compréhension du phénomène étudié. Le canal des participants a été mobilisé pour mieux comprendre le phénomène de structuration du CDG dans les PME. L'étude de cas multiple de type enchâssé a été adopté au regard de la spécificité de l'étude. Les entretiens au sein des PME retenues ont été menés auprès de plusieurs personnels en fonctions dans le souci de mieux comprendre les tâches et le fonctionnement et le système d'information. L'étude de cas est pertinente pour décrire et comprendre avec précision les phénomènes au regard de la spécificité de la problématique de recherche et de la nature de l'objet étudié. Ce cadre d'analyse nous offre la flexibilité nécessaire pour répondre au double objectif de description et représentation théorique dans ce travail. La pertinence de la méthode de cas réside dans leur capacité à aller en profondeur pour mettre en lumière les singularités d'un objet d'étude complexe dont les méthodes classiques trouvent des limites.

\subsection{La sélection des différents cas de l'étude}

Le choix de l'objet de l'étude s'est fait sur la base d'un certain nombre de critères. Les PME qui ont formalisé le CDG afin d'étudier leurs particularités et éléments de contexte ont rendu cela possible. L'objectif de cette étude est d'analyser les mécanismes de structuration du CDG dans les entreprises et de dégager les principaux facteurs. Les dimensions de l'observation sont donc multiples. De plus, la collecte des données a été orientée de manière à reconstituer les événements à posteriori. 
Nous avons également mis à contribution notre répertoire de contacts et le fichier des contribuables disponibles sur le site des impôts répertoriés un certain nombre d'entreprise devant faire partie de notre échantillon théorique soit près de 20 entreprises.

Les critères de sélection de notre échantillon de départ portent sur l'existence d'une fonction CDG ou DAF avec un effectif important et l'appartenance à la catégorie des PME (chiffre d'affaires, effectif du personnel, ou encore indicateur qualitatif). Sur les 20 PME de départs, 12 entreprises respectaient nos critères de sélection, mais seulement 8 étaient disposées de nous fournir les informations nécessaires à la bonne compréhension du phénomène étudié.

\subsection{La techniques d'investigation et l'analyse des données}

Nos recherches empiriques ont été menées entre 20 juin 2019 et 25 juin 2020 (dans sa phase d'analyse qualitative). La longueur de ce processus peut s'expliquer par le fait que nous nous déplacions entre la théorie, le terrain et l'analyse. Les études de cas réalisées s'appuient sur une mission qui est de rendre compte de la structuration du CDG dans les PME. L'exploitation de plusieurs sources tels que les documents officiels (plan stratégique, plans d'action, manuels et procédures, rapport d'activité, états physiques de CDG, ...), les entretiens semi-directifs avec des acteurs principaux du CDG (le DAF mis pour Directeur Administratif et Financier ou Responsable Administratif et financier, le contrôleur de gestion). Afin de déterminer tous les contours du problème, les faits observés dans cette étude sont confrontés et d'autres expressions (notamment à travers des entretiens avec plusieurs acteurs de l'entreprise). Nous avons utilisé deux logiciels fréquemment mobilisés dans l'analyse des données qualitatives. II s'agit du logiciel Atlas_ti_8 qui permet une gestion des codes aisée afin de former des catégories conceptuelles et du logiciel QDA NER indiqué pour des analyses de données textuelles mixtes "qualitative-quantitative ". La démarche d'analyse empirique se décline en quatre étapes à savoir : l'information textuelle, l'unité de codage, la fréquence des codes et la co-occurrence des codes et des cas. S'agissant de la présentation des résultats, les verbatim sont cités dans leur première version, sans retouche de notre part afin de préserver l'authenticité du propos. Les caractéristiques des PME faisant partie de notre échantillon sont consignées en annexe.

\section{Les résultats de l'étude}

Au terme de l'analyse des contenus thématiques, la démarche de structuration du CDG, les outils mobilisés et les différents acteurs intervenants se présentent comme suit.

\subsection{La démarche de structuration du CDG dans les PME camerounaises}

Nous avons mis en évidence dans les corpus analysés les différents stades de développement. Ceuxci sont spécifiquement liés à la culture de gestion précédente ou l'état initial à faire évoluer, le changement dans la structure organisationnelle et dans l'instrumentation. Mettre en évidence ces aspects permet de vérifier l'existence d'une éventuelle structuration du CDG dans l'entreprise. En d'autres termes, il s'agit de voir d'où l'entreprise est partie est à quel stade elle se trouve dans sa démarche de structuration. Nous avons aussi dégagé les perspectives dans l'instrumentation de gestion des entreprises étudiées.

\subsubsection{Une culture de gestion à prédominance informelle}

Trois situations majeures sont en évidence: 
- La prédominance de l'informel dans la gestion de l'entreprise : de manière générale c'est cette situation qui prévaut et cela est clairement visible à l'analyse des Cas1, 3, 4 et 7. On constate aucune procédure n'est écrite, aucune organisation n'est structurée (absence d'organigramme, de fiche de poste, ...). Il y règne une cacophonie où le dirigeant a la main mise sur la gestion dans ces différents aspects. Par contre, dans le Cas2, nous avons noté qu'une procédure écrite existe. Ce qui différencie cette entreprise des autres cas analysés.

- La perte de confiance dans les informations de gestion produites : Cette situation est davantage visible avec le Cas 6 . "A chaque fois qu'il obtient une information de son service comptable lorsqu'il cherchait à travailler avec ses partenaires, il existait toujours des différences qu'il avait du mal justifiées ».

- La perte de contrôle de l'activité : «Cette situation découle dans les différents Cas 1, 3, 4, 5 et 8 de la gestion informelle de l'entreprise telle que mise en évidence précédemment. Elle a pour conséquence, la récurrence des vols, les pertes diverses dans l'entreprise et le sentiment d'impuissance du dirigeant. Celui-ci semble en effet perdre la main sur son activité. Nous notons par exemple dans le Cas3 que l'entreprise a des soucis dans ses rapports avec l'administration fiscale compte tenu des retards accumulés dans les déclarations, un stock géré sur fiche manuelle dont elle semble perdre la main. Au niveau du Cas6, le dirigeant face à l'absence d'une information crédible se fait du souci pour son patrimoine ".

\subsubsection{Le changement dans la structure de l'entreprise}

De l'analyse des corpus des différents cas, on note des aménagements au niveau de la structure hiérarchique de l'entreprise. Deux cas de figure se présentent dans les entreprises étudiées :

- La création d'une nouvelle fonction (DAF ou CDG) avec la dotation en ressource soit par recrutement externe ou promotion en interne d'une ressource dont le top management estime avoir le profil. Dans le Cas3 à la création la direction de l'entreprise a affecté deux personnels, l'un a bénéficié d'une promotion en interne et l'autre a été débauché d'un cabinet compte tenu de ses compétences recherchées.

- La modification apportée à une fonction existante : c'est la situation des Cas 2 et 8, initialement il s'agit d'une fonction de contrôleur et non de CDG. Plusieurs mois après le contrôleur s'engage véritablement à apporter les aménagements à cette fonction pour introduire le CDG. L'intérêt est plus ressenti au niveau du Cas 8 , le contrôleur de gestion nous révèle qu' " en tant que contrôleur mes actions étaient très limitées à quelques aspects de l'activité. Je n'avais pas le contrôle complet de la société. J'étais un contrôleur qui regardait juste le système de vente des hébergements, la restauration, les stocks physiques, ... les autres éléments je n'étais pas intéressé » (Répondant Cas8).

\subsubsection{Le changement dans l'instrumentation}

Il porte sur les nouveaux procédés de travail ainsi que les outils mobilisés.

- Mise en place d'un système d'information : De part et d'autre, où il n' existait aucun instrument informatique, l'entreprise se dote des logiciels de gestion pour améliorer son système d'information. C'est la première étape pour sortir progressivement de l'informel. La gestion des stocks et la comptabilité ne sont plus tenues manuellement avec les conséquences relevées précédemment. 
- La dotation de l'entreprise des nouveaux outils : On note que la structuration du CDG vient avec de nouveaux outils, les plus récurrents sont par exemple les budgets, tableaux et rapports. Et avec le système informatique qui s'est amélioré, nous avons des outils standards édités directement dans le logiciel. Par exemple, le répondant du Cas8 nous révèle qu' "il y a les tableaux de bord fournis par le logiciel » (Répondant Cas8). Ceci est valable pour tous les cas étudiés.

La périodicité de traitement des informations s'est vue aussi s'améliorer. S'il fallait attendre un mois, la présence d'un contrôleur de gestion ou DAF a permis à l'entreprise suivre certains aspects de l'activité au jour le jour. Ce point est plus marqué chez le Cas7. Avec le Cas3, nous avons noté I'acquisition d'un ERP dans sa démarche de structuration du CDG. Cet investissement a permis à l'entreprise d'interconnecter ses agences avec sa direction et rendre l'information basique (charges, chiffre d'affaires, stocks, ...) disponible de façon instantanée.

\subsection{La dynamique acteurs/outils de la démarche de structuration du CDG}

L'analyse de la dynamique acteurs/outils dans le processus étudié met en évidence les acteurs de la structuration et les étapes suivies.

\subsection{1- Les acteurs de la structuration}

L'analyse des corpus a permis de mettre en évidence les acteurs internes et ceux externes à l'entreprise.

\section{- Les acteurs internes}

Les acteurs internes dans le processus par ordre d'importance sont, les dirigeants $(24,9 \%$ d'occurrences), les opérationnels (22,45\% d'occurrences), les contrôleurs de gestion $(20,41 \%$ d'occurrences), les comptables (18,37\% d'occurrences) et en dernier les DAF (14,29\% d'occurrences). Selon les cas, les acteurs internes sont différents. Mais de manière générale les dirigeants et les opérationnels ont plus d'impact dans le processus que même les contrôleurs de gestion ou les DAF. Toutefois, il faut relever que les contrôleurs de gestion et les DAF sont les acteurs majeurs dans ce sens que si on cumule leurs taux occurrences ces acteurs détiendront le premier rang avec $34,7 \%$ ce qui les placent largement en tête des acteurs internes.

\section{- Les acteurs externes}

De l'analyse des corpus tirés du terrain, les acteurs externes cités par ordre d'importance sont les experts comptables/Cabinets (73,68\% d'occurrences) et les consultants indépendants $(26,32 \%$ d'occurrences). L'expert-comptable/Cabinet a une plus grande influence sur la démarche de manière générale par rapport aux consultants indépendants. Le Cas 2 est assez particulier, aucun acteur externe ne se démarque véritablement. Une fois, que les acteurs qui ont une importance dans le processus ont été mis en évidence, nous nous intéressons à présent aux étapes suivies par le processus dans les PME camerounaises.

\subsubsection{Les outils mobilisés dans le processus}

Afin d'identifier les outils de CDG utilisés dans les PME, nous avons minutieusement analysé les corpus. L'outil est un instrument utilisé par la PME qui sert de base de travail ou de modification de certaines 
configurations des activités de l'entreprise et des comportements en son sein. La description se fera en allant des outils les plus récurrents (plus cités) vers ceux qui le sont moins (moins cités).

- parmi les outils les plus récurrents (récurrence plus élevées), nous pouvons citer par ordre d'importance les inventaires, les objectifs, les rapports divers, la comptabilité, les budgets, le manuel des procédures et les tableaux (tableaux de bord, tableaux divers, ...) ;

- parmi les outils à récurrence moyenne, nous pouvons citer par ordre d'importance les différents états, diverses fiches, le calcul des coûts, la tenue des réunions, les marges et les logiciels à I'instar de SAGE SAARI plus répandu. Quand nous évoquons le terme « logiciel » ici, il faut voir les états standards produits par les différents logiciels sur certains aspects de l'activité. Le répondant du Cas8, nous révèle par exemple qu' " il y a les tableaux de bord fournis par le logiciel assez sommaire qui ne sont pas complet. Cela ne prend en compte que les éléments d'exploitation directe " (Répondant du Cas8). Les états produits par le logiciel appellent encore d'autres traitements ou outils complémentaires ;

- parmi les outils avec une récurrence faible ou quasi nulle : nous pouvons citer par ordre d'importance les ratios, l'organigramme, les standards, l'ERP, les journaux principalement de caisse et de vente, le compte de résultat/Bilan, le brouillard de caisse et les seuils.

\subsection{Les caractéristiques de la démarche de structuration du CDG dans les PME camerounaises}

L'analyse des corpus obtenus nous permet d'identifier plusieurs caractéristiques du processus de structuration du CDG dans les PME camerounaises.

Cette analyse s'appuie sur l'exploitation des co-occurrences entre les cas et les différentes propriétés mises en évidence à savoir, les outils, les types de fonctions, les acteurs de la démarche, les étapes du processus. Nous obtenons au regard des relations potentielles entre les variables, la similarité parmi les cas, qui se constituent en 3 grands groupes:

\section{Figure $n^{\circ} 1$ : Le dendrogramme de similarités des cas à trois classes}

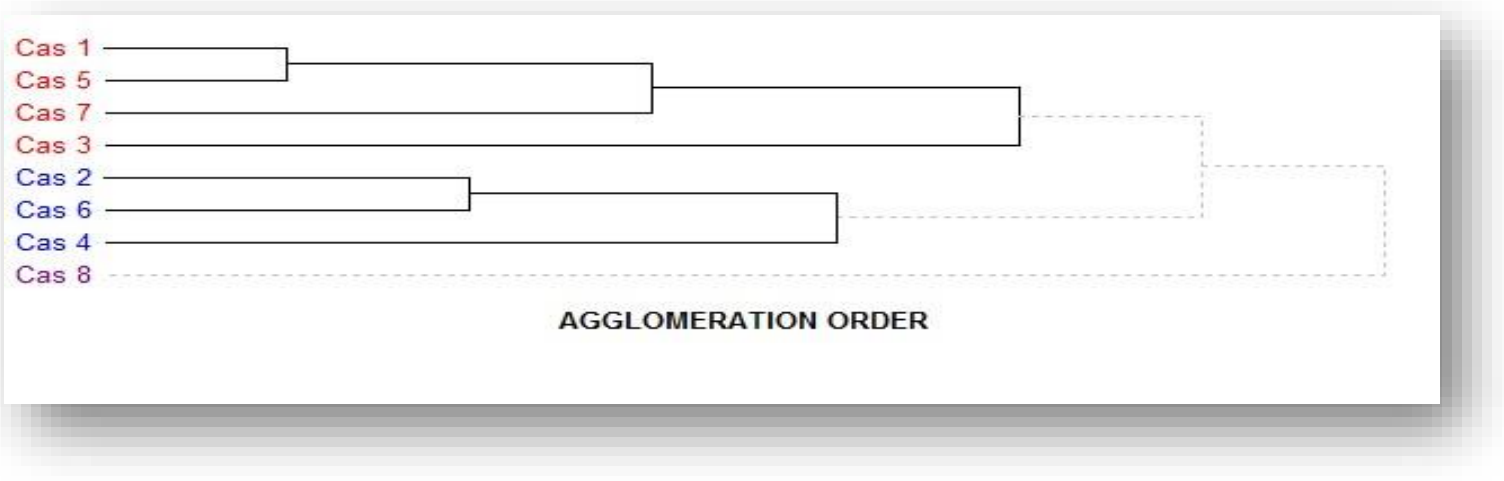

\section{Source : QDA Miner v5}

Pour chacun des groupes, nous avons identifié la démarche de structuration du CDG associée (Voir tableau $n^{\circ} 2$ ci-dessous). II se dégage que le Groupe 1 est principalement associé à la démarche 1 de structuration du CDG. Le Groupe 2 regroupe 3 cas étudiés (cas4, cas2 et cas6). Ce groupe est associé à la démarche 2 de structuration du CDG. Le dernier groupe (le groupe 3) est lié à la démarche 3. 


\section{$\underline{\text { Tableau n }{ }^{\circ} \text { : }}$ Les caractéristiques du processus de structuration du CDG dans les PME} camerounaises.

\begin{tabular}{|c|l|c|}
\hline $\begin{array}{c}\text { Caractéristiques } \\
\begin{array}{c}\text { Processus type } \\
\text { progressif }\end{array}\end{array}$ & $\begin{array}{l}\text { Il s'agit d'un processus dont les propriétés sont évolutives au cours du temps } \\
\text { et dont les perspectives sont perceptibles. }\end{array}$ & $\begin{array}{c}\text { Cas1; Cas3 ; Cas5; } \\
\text { Cas7 }\end{array}$ \\
\hline $\begin{array}{c}\text { Processus type } \\
\text { stationnaire }\end{array}$ & $\begin{array}{l}\text { Il s'agit ici d'un processus dont les propriétés restent inchangées au cours } \\
\text { du temps et dont les perspectives d'évolution existent mais ne sont pas } \\
\text { concrétisées. }\end{array}$ & Cas4; Cas2; Cas6 \\
\hline $\begin{array}{c}\text { Processus type } \\
\text { endormi ou bloqué }\end{array}$ & $\begin{array}{l}\text { Il s'agit ici d'un processus dont les propriétés ont atteint leur point optimal } \\
\text { et dont les possibilités de développement sont quasi absentes. }\end{array}$ & Cas8 \\
\hline
\end{tabular}

\section{Source : Auteur}

D’une manière générale, la démarche de structuration présente deux configurations majeures soit elle est progressive soit elle est stationnaire. Un processus bloqué ne présentant plus un réel intérêt.

\subsection{Les étapes de structuration de structuration du CDG dans les PME}

Les différentes démarches de structuration du CDG qui se dégagent de l'analyse des différents cas de notre étude se présentent comme suit.

\section{Tableau n 03: Démarche de structuration du CDG}

\begin{tabular}{|c|c|c|c|}
\hline Concept théorique & Etape du processus & Acteurs & $\begin{array}{c}\text { Principaux Outils } \\
\text { mobilisés }\end{array}$ \\
\hline Problématisation & $\begin{array}{l}\text { ETAPE1 (E1) } \\
\text { E.1.1.Problématisation de la situation par } \\
\text { certains acteurs stratégiques } \\
\text { E.1.2. Remise en cause du pilotage intuitif } \\
\text { E.1.3.Mise en évidence des failles et } \\
\text { insuffisances dans le fonctionnement }\end{array}$ & $\begin{array}{c}\text { Le Dirigeant } \\
\text { Professionnels externes } \\
\text { (Expert-comptable, } \\
\text { consultants, ...) }\end{array}$ & $\begin{array}{l}\text { Outils réglementés } \\
\text { (quelques outils } \\
\text { principaux) }\end{array}$ \\
\hline Enrôlement & $\begin{array}{l}\text { ETAPE2 (E2) } \\
\text { E.2.1.Recrutement du DAF et/ou du } \\
\text { contrôleur de gestion } \\
\text { E.2.2.Promotion interne d'un profil avec } \\
\text { des aptitudes supposées } \\
\text { E.2.3.Attribution de missions techniques } \\
\text { au CDG }\end{array}$ & $\begin{array}{l}\text { - Le Dirigeant } \\
\text { - Professionnels externes } \\
\text { (Expert-comptable, } \\
\text { Consultants, ...) } \\
\text { - DAF et/ou Contrôleur de } \\
\text { gestion (nouvel acteur) }\end{array}$ & $\begin{array}{l}\text { Outils financiers + } \\
\text { Outils réglementés } \\
\text { améliorés }\end{array}$ \\
\hline \multirow{2}{*}{$\begin{array}{l}\text { Mobilisation } \\
\text { \& } \\
\text { Intéressement }\end{array}$} & $\begin{array}{l}\text { ETAPE3 (E3) } \\
\text { E.3.1.Mobilisation des autres acteurs } \\
\text { autours des outils de CDG (existants et } \\
\text { nouveaux) } \\
\text { E.3.2.Intéressement des autres acteurs } \\
\text { autour de la motivation, des } \\
\text { encouragements divers, ... }\end{array}$ & $\begin{array}{l}\text { - Le Dirigeant } \\
\text { - DAF et/ou Contrôleur de } \\
\text { gestion } \\
\text { - le comptable } \\
\text { - Les autres responsables de } \\
\text { l'entreprise }\end{array}$ & $\begin{array}{l}\text { Outils financiers + } \\
\text { Outils réglementés } \\
\text { améliorés + Outils } \\
\text { standardisés }\end{array}$ \\
\hline & $\begin{array}{l}\text { ETAPE4 (E4) } \\
\text { E.4.1.Amélioration du système } \\
\text { d'information (Acquisitions des ERP, ...) } \\
\text { E.4.2. Décentralisation des contrôleurs de } \\
\text { gestion dans les différentes entités avec } \\
\text { un poste central au siège }\end{array}$ & $\begin{array}{l}\text { - Le Dirigeant } \\
\text { - DAF et/ou Contrôleur de } \\
\text { gestion }\end{array}$ & $\begin{array}{c}\text { Outils financiers + } \\
\text { Outils réglementés } \\
\text { améliorés + Outils } \\
\text { standardisés améliorés }\end{array}$ \\
\hline
\end{tabular}

\footnotetext{
Source : Auteur
} 
Nous constatons qu'effectivement la structuration du CDG dans les PME s'effectue par étape.

- Le processus de structuration du CDG dans les PME s'amorce par la problématisation des difficultés majeures de gestion à résoudre.

- Le processus de structuration du CDG dans les PME procède d'un changement dans la structure de l'entreprise à travers la création de fonctions spécialisées et le recrutement de spécialistes.

- Le processus de structuration du CDG dans les PME aboutit à un changement dans I'instrumentation de gestion ou un statu quo.

\subsection{Discussion des résultats}

La discussion autour des résultats va porter sur les caractéristiques de la démarche, les acteurs et les facteurs.

\subsubsection{L'analyse du processus de structuration du CDG}

La structuration du CDG dans les PME s'effectue en 4 étapes de manière générale. La phase de la problématisation de la situation par le dirigeant, la phase d'enrôlement d'un nouvel acteur ou d'attribution de nouvelle fonction à un acteur interne à l'entreprise et deux étapes alternées de mobilisation et d'intéressement. Les dernières étapes consistent à la mobilisation des autres acteurs de l'entreprise autour des nouvelles méthodes et outils de CDG.

Au cours de cette étape l'amélioration du système d'information avec les acquisitions des nouveaux logiciels de gestion. L'étape d'intéressement n'est pas partagée par plusieurs entreprises, parce que dans le contexte camerounais, elle consiste en la motivation et l'encouragement des autres acteurs à s'intéresser au processus. Au cours de ces phases la fonction CDG est décentralisée dans les entités de l'entreprise. Il se passe que chaque entité a son contrôleur de gestion et un poste contrôleur de gestion central est créé au siège. Un rapprochement fait avec les travaux de Barbelivien (2016), permet de mettre en évidence plusieurs points de divergences bien qu'il existe quelques points de convergence. Nous analysons en premier les points de convergence entre les travaux de Barbelivien (2016a), et nos résultats. En effet, nous parvenons à la même conclusion selon laquelle la démarche de structuration du CDG dans les PME se fait par étapes successives. Cette conclusion est aussi conforme aux résultats de Barbelivien et Meyssonnier (2018). Ces derniers affirmaient que la structuration du CDG est le passage d'un point to à un point t1 avec des améliorations dans l'instrumentation de gestion. Par ailleurs, la démarche de structuration dans les PME camerounaises comme dans les travaux de Barbelivien (2016a) commence par une étape de la problématisation d'une situation de gestion inconfortable. Ici le dirigeant est l'acteur principal, dans les PME camerounaises, il est quelques fois aidé par des professionnels conseils.

Dans une seconde analyse, les points de divergences qui se dégagent dans le rapprochement de nos résultats avec ceux de Barbelivien (2016) portent sur la longueur des étapes. II dénombre 6 étapes dans notre contexte nous recensons 4 étapes. La démarche est moins structurée qu'ailleurs, contrairement à la démarche de Barbelivien (op cité), il n'existe pas de test pilote des outils introduits dans la démarche. Les outils sont introduits les uns après les autres s'arrangent à se les approprier. II existe une phase d'intéressement des autres acteurs dans le processus juste après l'étape de problématisation ce qui n'est pas le cas au terme de notre analyse. En effet, chez Barbelivien (2016a), 
les autres responsables sont impliqués dès le début du projet, il est d'ailleurs élaboré avec leurs concours et ils sont informés de la démarche ; contrairement à chez nous où le dirigeant une fois que la solution est toute trouvée dans sa tête il prend des initiatives et les autres responsables sont mis devant les faits accomplis. Ce qui quelques fois est dommage parce qu'il met ses collaborateurs dans des situations très inconfortables. Ceux-ci auront à faire à de nouvelles méthodes de travail et de nouveaux outils dont ils n'ont pas été préparés. C'est ce qui est souvent à l'origine des réticences et frustrations que nous avons pu apprécier sur le terrain.

Contrairement à Barbelivien (2016a) nous avons identifié trois caractéristiques du processus progressif, stationnaire et endormi ou bloqué. Nous avons en effet, remarqué qu'il existe des configurations où le processus n'est pas abouti mais tout semble être comme à l'arrêt, les perspectives d'évolution existent mais ne sont pas d'actualité et même les acteurs principaux n'ont aucune lisibilité sur cela. Nous sommes là dans un processus type stationnaire. Nous avons néanmoins noté plusieurs entreprises qui s'inscrivent dans une démarche progressive de structuration dont le processus suit son cours.

\subsubsection{L’analyse des différentes implications des résultats}

Dans cette rubrique nous passerons en revue les différentes implications théoriques et managériales de notre travail.

\section{Inscrire le processus de structuration du CDG dans le cadre d'un projet}

L'analyse des travaux sur le sujet et les résultats issus du terrain permettent de relever que la structuration du CDG dans les PME, procède par étapes. Ces étapes peuvent selon les contextes (pays occidentaux, ou pays de l'Afrique Subsaharienne) être différentes. Pour le mener à bien compte tenu de son importance, sa sensibilité (du fait l'influence des acteurs), du temps long, ... il apparaît qu'inscrire la démarche dans le cadre d'un projet sérieux semble pertinent. Dans ce sens qu'un projet regroupe l'ensemble d'activités organisées en phases ou étapes et formant l'unité de gestion permettant la réalisation d'un objectif défini et précis. En effet, le besoin de structuration du CDG dans la PME obéît à la recherche d'un objectif précis qui se dessine au moment de la problématisation.

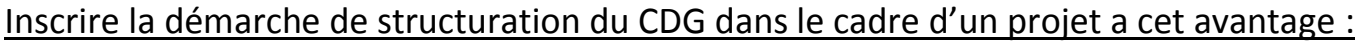

- l'objectif est partagé ;

- identifier et impliquer les acteurs ;

- les coûts évalués (acquisitions des ressources humaines et matérielles nécessaires au projet) ;

- définir les délais (courts, moyen, long terme, ...) ;

- définir les étapes du projet et les livrables attendus ;

- une large communication et implication au tour du projet ;

- il permet de gérer les changements pouvant survenir du fait de l'implémentation du projet ;

Ainsi, la gestion de la structuration du CDG en tant que projet, va consister à organiser et suivre chaque action du projet afin de tenir les délais et les attentes. En la matière un cahier de charge du projet avec l'appui des partenaires conseil plus expérimentés pourra être dressé pour aider l'entreprise à savoir quel est le point de départ et quel est son point de chute. 


\section{Une période nécessaire pour une transition}

Le projet de structuration du CDG une fois arrêté ainsi que la vision associée et les participants identifiés, il devrait y avoir une période de transition au cours de laquelle une culture de gestion est développée puis complétée par les outils de CDG. Cette période de transition permet l'introduction graduelle des outils de gestion à partir de la mise en place des groupes de travail, des comptes rendus de réunions et des rapports d'activités. L'idée est d'éviter un changement brusque dans l'entreprise, mais d'amener progressivement les acteurs de l'entreprise à intégrer les changements à opérer, à se préparer en conséquence mais surtout de participer à travers toutes les plateformes de concertation à mettre en place pour suivre le projet. Le dispositif apparaît dès lors non pas comme quelque chose d'imposer mais de conciliant auquel chaque acteur aura pris part pour sa construction. L'entreprise dispose alors d'un temps à sa convenance pour préparer les salariés au changement. Le temps nécessaire à la réalisation d'un projet de structuration est fonction des entreprises. Les réalités internes étant différentes (technologies, attentes, capacités financières, ...), il est quasi impossible de préconiser le temps nécessaire à la réalisation d'un tel projet. Toutefois, il est souhaitable que la période transitoire ne puisse pas excéder 1 (un) an suivant les contextes et les enjeux stratégiques de l'entreprise.

Après avoir émis des préconisations quant aux situations de gestion, nous allons à présent nous intéresser aux acteurs et formuler des recommandations permettant d'encourager leur implication, gérer la résistance au changement et favoriser l'appropriation de la démarche.

\section{Conclusion}

L'objectif de ce travail est d'analyser le processus par lequel les PME structurent leurs fonctions CDG. Pour y parvenir nous avons mobilisé une approche méthodologique qualitative. Elle a consisté à une étude de huit (08) cas de PME camerounaises. Au terme de l'analyse des données recueillies, nous avons abouti aux résultats selon lesquels la structuration du CDG dans les PME est un processus qui s'effectue par étape successive la première étape majeure du processus est celle de la problématisation d'une difficulté de gestion par le dirigeant quelques fois appuyé par des professionnels conseils (experts comptables, consultants, cabinets, ...). La seconde étape majeure est celle du changement dans la structure de l'entreprise. Ici l'entreprise procède à la création de nouvelles fonctions spécialisées (DAF, CDG, ...). Le dirigeant ou le comptable qui cumulait plusieurs rôles se voit décharger des rôles superflus pour les confier aux spécialistes. L'étape déterminante à laquelle le processus doit aboutir est le changement dans l'instrumentation de gestion. Le processus est soit de type progressif, soit de type stationnaire, ou soit de type bloqué. Chaque type de processus a ses particularités. Mais dans le travail, les processus de type progressif et stationnaire ont été pertinent parce que présentant des perspectives d'évolution. Ce qui n'est pas le cas d'un processus de type bloqué. Sur la base de ces résultats, notre étude aboutit aux recommandations selon lesquelles le processus de structuration doit s'inscrire dans le cadre d'un projet bien structuré. Cette étude permet de mieux cerner les contours du processus de formalisation de la fonction CDG dans les entreprises, principalement celles camerounaises. 


\section{BIBLIOGRAPHIE}

[1] Akrich, M., Callon M. \& Latour, B., (1988) : A quoi tient le succès des innovations. Deuxième épisode : I'art de choisir les bons porte-parole ", Annales des Mines.

[2] Alcouffe, S., Berland, N. \& Levant, Y. (2008). " Actor-networks and the diffusion of management accounting innovations : a comparative study ", Management Accounting Research, Vol.19, $n^{\circ} 1$, pp. 1-17.

[3] Barbelivien, D. \& Meyssonnier, F. (2018). "Une contribution à l'étude de la structuration du contrôle de gestion en PME : le cas de trois entreprises familiales et industrielles de taille intermédiaire (ETI)». Revue internationale P.M.E., Vol. $31 \mathrm{n}^{\circ} 1$, pp. 157-185. https://doi.org/10.7202/1044692ar

[4] Dupuy, J.P., Eymard-Duvernay, F., Favereau, O., Orléan, A., Salais, R., \& Thévenot, L., (1989). "Introduction au numéro spécial de la revue économique sur l'économie des conventions ", Revue Economique, vol. 40, n² 2, mars, pp. 141-145.

[5] Gomez, P.Y. (1996). "Information et convention ; le cadre du modèle général ", Revue Française de Gestion, $n^{\circ} 112$, janvier-février, pp. 64-77.

[6] Marchesnay, M. et Fourcade, C. (1997) : Gestion de la PME/PMI, Nathan.

[7] Mayéglè, F-X. \& Ngo Nguidjol, S. (2016) : "Déterminants du choix du système de contrôle de gestion dans les PME camerounaises : une approche contingente ", 1ère Journée d'Etude Africaine en Comptabilité et Contrôle

[8] Meyssonnier, F. \& Zawadzki, C. (2008) : «L'introduction du contrôle de gestion en PME : étude $d^{\prime} u n$ cas de structuration tardive de la gestion d'une entreprise familiale en forte croissance ", Revue Internationale PME, Vol.21, n¹, pp. 69-92.

[9] Montmorillon, B. (1999). "Théories des conventions, rationalité mimétique et gestion d'entreprise ", in G. Koenig (éd.), De nouvelles théories pour gérer l'entreprise du XXI ème siècle, Paris, Economica, 1999, p. 171-198.

[10] Ngongang, D., (2013). "Système d'information comptable et contrôle de gestion dans les entreprises camerounaises ", DOI: 10.1016/j.rgo.2013.12.005

[11] Nobre T. (2001a), "Le contrôleur de gestion en PME ", Comptabilité Contrôle Audit, mars, tome 7, vol 1, pp. 129-146.

[12] Simons, R. (1995). Levers of Control. How Managers Use Innovative Control Systems to Drive Strategic Renewal, Harvard Business School Press, Boston, 217 p.

[13] Zawadzki, C. (2009) : Enjeux et difficultés de l'introduction du contrôle de gestion : une étude de cas en pme, Thèse de doctorat, Université Paul Verlaine - Metz soutenue le 7 juillet, 264 pages. 
Annexe : Les caractérisques des PME interviewées

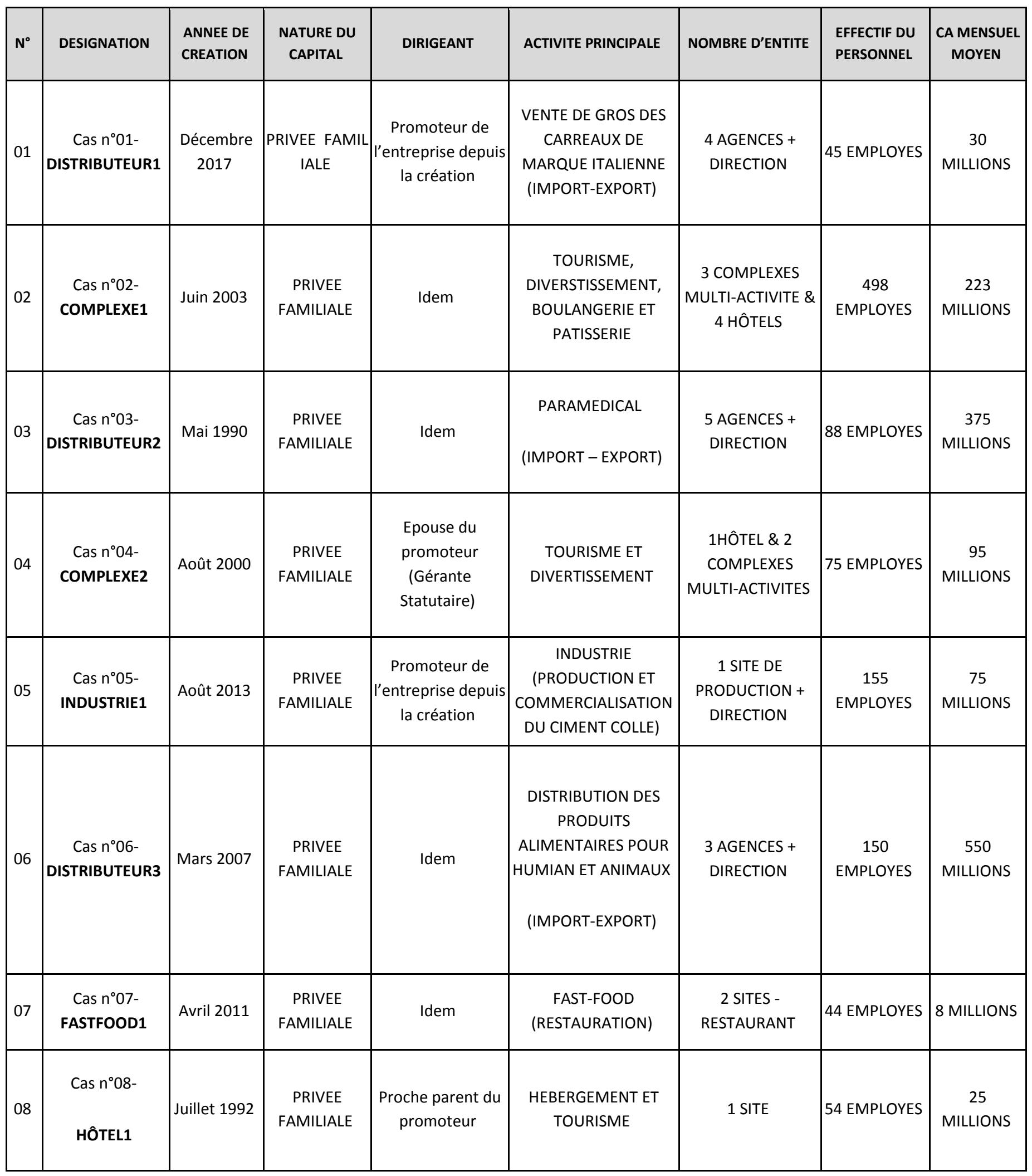

